\title{
Threshold Effects in Three-Terminal Hybrid Systems
}

\author{
G. MiCHAEEK*
}

Institute of Molecular Physics, Polish Academy of Sciences ul. Mariana Smoluchowskiego 17, 60-179 Poznań, Poland

\begin{abstract}
In the paper we study transport in the three terminal hybrid devices with quantum dot. Our attention is focused on the conductance threshold effects (so called Wigner singularities) caused by the back action from the side-wire. In particular we study influence of the direct electron transfer (ET) and crossed Andreev reflection (CAR) processes on the conductance caused by the direct Andreev reflection (DAR) processes.
\end{abstract}

DOI: $10.12693 /$ APhysPolA.126.224

PACS: 73.63.Kv, 73.63.-b, 74.45.+c

\section{Introduction}

The devices which consists of the quantum dot (QD) coupled with superconducting and normal leads are being extensively studied recently, because of their potential application in nanoelectronic, spintronic and quantum computation processing, see e.g. [1].

In this paper a quantum dot coupled to two semiinfinite one-dimensional wires (source-L and side-R) and one superconducting (drain-S) electrode is considered. Our attention is focused on the influence of the transmission into the side wire on the current flows between the source wire and the drain electrode. In particular, when a new conductance channel opens conduction in the side wire, one observes threshold effects in the conductance (so called Wigner singularities [2]), caused by the back action from this wire. The shape of the singularities depends on the interference conditions when the new conduction channel is activated. A detailed analysis of the direct electron transfer (ET) and the Andreev reflection (AR) contributions to the conductance allows one to identify the microscopic processes, which are responsible for the particular shape of the Wigner singularities.

\section{Description of the model and method of calculation}

We consider noninteracting electrons on the QD with only one single-particle energy level $\varepsilon_{0}$ available for tunnelling. The wires are treated within the tight-binding approximation for the semi-infinite chain of atoms [3] with hopping integrals $t_{\alpha}$ (taken as real) between neighbouring sites and site energies $\varepsilon_{\alpha}, \alpha=\{L, R\}$. The superconducting electrode is described in the BCS approximation. The bias voltage $V_{L}$ is applied only to the $\mathrm{L}$ wire, while the $\mathrm{R}$ wire and the $\mathrm{S}$ electrode are grounded. An additional gate potential $V_{G}$ applied to the $\mathrm{R}$ wire changes the position of the site energies $\varepsilon_{R}$, so that the energy bands for electrons and holes are shifted.

Using the equation of motion technique (EOM) for the non-equilibrium Green function one can find currents originating from various types of tunnelling processes $[4,5]$. In the subgap regime $\left|e V_{L}\right|<\Delta$ the total

*e-mail: grzechal@ifmpan.poznan.pl

current from the left electrode

$$
I_{L}^{T O T}=I_{L}^{E T}+I_{L}^{D A R}+I_{L}^{C A R},
$$

where ET denotes normal electron tunnelling and DAR (CAR) describes direct (crossed) Andreev reflection.

$$
\begin{aligned}
& I_{L}^{E T}=\frac{2 e}{\hbar} \int \frac{d E}{2 \pi} \Gamma_{L}^{e}\left|G_{11}^{r}\right|^{2} \Gamma_{R}^{e}\left(f_{L}^{e}-f_{R}^{e}\right), \\
& I_{L}^{D A R, C A R}=\frac{2 e}{\hbar} \int \frac{d E}{2 \pi} \Gamma_{L}^{e}\left|G_{12}^{r}\right|^{2} \Gamma_{L, R}^{h}(E)\left(f_{L}^{e}-f_{L, R}^{h}\right) . \\
& f_{\alpha}^{e} \equiv f_{\alpha}^{e}(E)=1 /\left\{\exp \left[\left(E-e V_{\alpha}\right) / k_{B} T\right]+1\right\} \\
& f_{\alpha}^{h}=1-f_{\alpha}^{e}(-E)
\end{aligned}
$$

The electron and hole transfer between the QD and the $\mathrm{L}(\mathrm{R})$ wire is described by the effective tunnelling rates $\Gamma_{\alpha}^{e}=\frac{\gamma_{\alpha}}{2} \sqrt{4 t_{\alpha}^{2}-\left(E-\varepsilon_{\alpha}\right)^{2}}$ and $\Gamma_{\alpha}^{h}=$ $\frac{\gamma_{\alpha}}{2} \sqrt{4 t_{\alpha}^{2}-\left(E+\varepsilon_{\alpha}\right)^{2}}$ respectively. $\gamma_{\alpha}=2 t_{\alpha 0}^{2} / t_{\alpha}^{2}$, where $t_{\alpha 0}$ is the hopping integral between the QD and the $\alpha$ wire. $\Gamma_{S}$ is described in the wide band approximation, i.e. $\Gamma_{S}=2 \pi\left|t_{S}\right|^{2} \rho_{S}$, where $\rho_{S}$ is the density of states in the $\mathrm{S}$ electrode in the normal state. The Green functions (in the limit $\Delta \rightarrow \infty$ ) reads

$$
\begin{aligned}
G_{11}^{r} & =\frac{E+\varepsilon_{0}-\sum_{\alpha=L, R} t_{\alpha 0}^{2} g_{\alpha}^{h}}{\left(E-\sum_{\alpha=L, R} t_{\alpha 0}^{2} g_{\alpha}^{e}\right)^{2}-\varepsilon_{0}^{2}-\Gamma_{S}^{2} / 4}, \\
G_{12}^{r} & =\frac{1}{2} \frac{\Gamma_{S}}{E+\varepsilon_{0}-\sum_{\alpha=L, R} t_{\alpha 0}^{2} g_{\alpha}^{h}} G_{11}^{r},
\end{aligned}
$$

where $g_{\alpha}^{e}\left(g_{\alpha}^{h}\right)$ are Green functions for electrons (holes) in the wire $\alpha=\{L, R\}$.

Differential conductances are defined as

$$
\mathrm{G}_{L / L}^{\kappa}=\frac{d I_{L}^{\kappa}}{d V_{L}},
$$

where $\kappa=\{T O T, E T, D A R, C A R\}$.

\section{Results and conclusions}

As long as the gate voltage is not applied to the $\mathrm{R}$ wire, the electron and hole effective tunnelling rates are equal $\Gamma_{R}^{e}=\Gamma_{R}^{h}$, and are symmetrical functions with regard to the Fermi energy in the $\mathrm{R}$ wire. However, when an additional gate voltage is applied to the $\mathrm{R}$ wire, $\Gamma_{L}^{e}$ and $\Gamma_{L}^{h}$ 

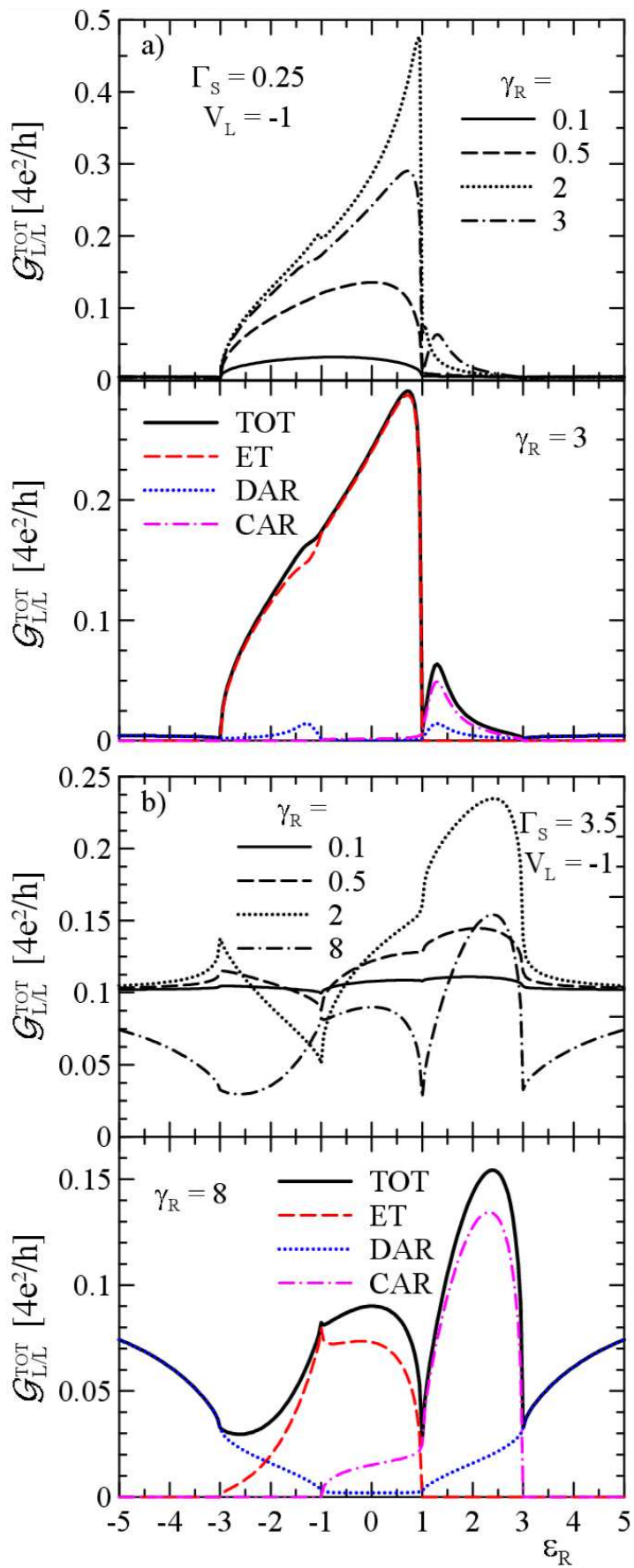

Fig. 1. $\mathrm{G}_{L / L}^{T O T}$ and its components for small $\Gamma_{S}<\gamma_{L}$ (a) and large $\Gamma_{S}>\gamma_{L}$ (b) vs. $\varepsilon_{R}=-e V_{G}$. The other parameters are $\gamma_{L}=0.5, t_{R}=1, \varepsilon_{0}=\varepsilon_{L}=0$, $e V_{L}=-1$ and $T=0$ in units of $t_{L}$ (taken as unity in the calculations).

are shifted in the opposite directions. In Fig. 1 the total conductance $\mathrm{G}_{L / L}^{T O T}$ and their components $\mathrm{G}_{L / L}^{E T}, \mathrm{G}_{L / L}^{D A R}$, and $\mathrm{G}_{L / L}^{C A R}$, in the case of weak and strong coupling to the $\mathrm{S}$ electrode (i.e. small and large proximity effect on the QD), are presented as a function of the gate voltage $\varepsilon_{R}=-e V_{G}$, which is swept across the electron and hole band edges. Opening of new conduction channels in the $\mathrm{R}$ wire activates an additional tunnelling processes: the direct electron transfer (ET) and crossed Andreev reflection (CAR). Therefore $\mathrm{G}_{L / L}^{T O T}$ can take various shapes at the band edges, which depend on the relationship between coupling to $\mathrm{R}$ and $\mathrm{S}$ terminals.

For small coupling $\Gamma_{S}<\gamma_{L}$ the DAR processes are weak, see Fig. 1a. At $\varepsilon_{R}=-3$, when the ET processes are activated, shape of the $G_{L / L}^{T O T}$ is dominated by the $\mathrm{G}_{L / L}^{E T}$ and does not change with $\gamma_{R}$. For $\varepsilon_{R}>-1$ also $\Gamma_{R}^{h} \neq 0$, so CAR processes play a role. The interplay between DAR, CAR and ET processes influences the shape of the Wigner singularities at $\varepsilon_{R}=-1$ and $\varepsilon_{R}=1$. In the latter case the $\Gamma_{R}^{e}$ vanishes and one observes a dip in the $\mathrm{G}_{L / L}^{T O T}$. For $\varepsilon_{R}>1$, the CAR processes dominate and are responsible for the shallow dip at $\varepsilon_{R}=3$.

When the coupling to the $\mathrm{S}$ electrode is strong enough, $\left(\Gamma_{S}>\gamma_{L}\right)$ one finds in the $\mathrm{G}_{L / L}^{T O T}$ the signatures of the Wigner cusp, inverted cusp, peaks, dips and saddle points, see Fig. 1b. When $\Gamma_{S}>\gamma_{L}+\gamma_{R}$ the $\mathrm{G}_{L / L}^{D A R}$ has a Wigner cusp at $\varepsilon_{R}=-3$, while the $\mathrm{G}_{L / L}^{E T}$ has a square root shape above this value. The interplay between DAR and ET processes results in the peak shape of the $G_{L / L}^{T O T}$ for small coupling $\gamma_{R}$ and in the cusp-like shape of the $\mathrm{G}_{L / L}^{T O T}$ for larger $\gamma_{R}$. Activation of the CAR processes at $\varepsilon_{R}=-1$ is responsible for the inverted cusp in the $\mathrm{G}_{L / L}^{T O T}$. When $\Gamma_{S}<\gamma_{L}+\gamma_{R}$, the $\mathrm{G}_{L / L}^{D A R}$ exhibits a saddle point at $\varepsilon_{R}=-3$, so one observes a dip in the $\mathrm{G}_{L / L}^{T O T}$, see Fig. $1 \mathrm{~b}$ for $\gamma_{R}=8$. The small peak at $\varepsilon_{R}=-1$ and the inverted cusp at $\varepsilon_{R}=1$ are due to activation and large enhancement of the CAR processes, respectively. The shape (saddle point or inverted cusp) of $\mathrm{G}_{L / L}^{T O T}$ at $\varepsilon_{R}=3$ is a result of the interplay of the CAR and DAR processes.

\section{Acknowledgments}

The author would to thank B.R. Bułka for stimulating and fruitful discussions. This work has been partially supported by the National Science Centre under the contract DEC-2012/05/B/ST3/ 03208 and by the EU project Marie Curie ITN NanoCTM.

\section{References}

[1] A. Martín-Rodero, A. Levy Yeyati, Advances in Physics 60, 899 (2011).

[2] B.R. Bułka, A. Tagliacozzo, Phys. Rev. B 79, 075436 (2009).

[3] E.N. Economou, Green's Functions in Quantum Physics, Springer-Verlag, Berlin 2006.

[4] H. Haug, A.-P. Jauho, Quantum Kinetics in Transport and Optics of Semiconductors, Springer Verlag, Berlin 2008.

[5] Q.-F. Sun, J. Wang, T.-H. Lin, Phys. Rev. B 62, 648 (2000). 